Supplementary Information for

\title{
Multi-Bit Analog Transmission Enabled by Electrostatically Reconfigurable Ambipolar and Anti-Ambipolar Transport
}

Kartikey Thakar and Saurabh Lodha

Department of Electrical Engineering, Indian Institute of Technology Bombay, Mumbai 400076, India.

*Email: slodha@ee.iitb.ac.in

\section{List of Sections}

S1. AFM data for $\mathrm{hBN}$ and $\mathrm{WSe}_{2}$ flakes

S2. Polarity transition in similar devices with ambipolar $\mathrm{WSe}_{2}$ and $\mathrm{MoSe}_{2}$ channels

S3. Output characteristics, estimation of interface trap density and scope for device performance improvement

S4. Transfer curves by interchanging $V_{C G}$ and $V_{B G}$

S5. Photoresponse of the device for a wide range of optical power ( $1 \mathrm{nW}$ to $1 \mu \mathrm{W})$

S6. FFT Analysis of the ac waveforms

S7. Hysteresis and variability in device operation

S8. Switching data at $1 \mathrm{kHz}$ signal for anti-ambipolar mode of operation

S9. Left shift in anti-ambipolar peak under illumination

S10. Real-time ac switching showing a frequency-shift with the laser

S11. Metrics and $E$ values for 2-bit schemes

S12. Performance evaluation of 3-bit schemes

S13. Performance evaluation for a symbol space of 20 points 
S1: AFM data for $\mathrm{hBN}$ and WSe2 flakes

(a)

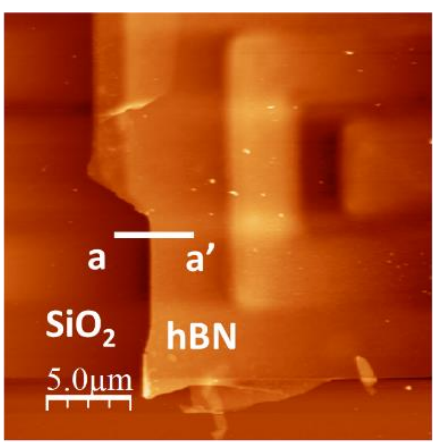

(c)

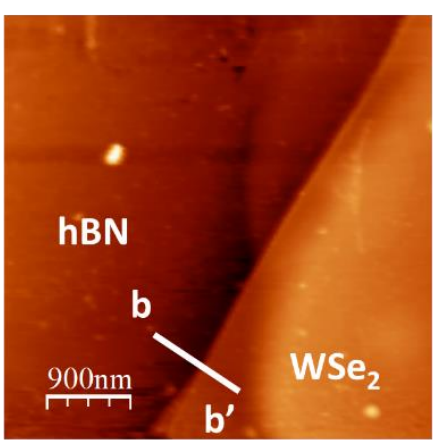

(b)

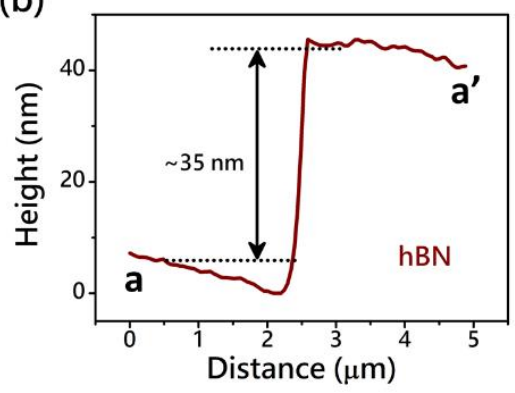

(d)

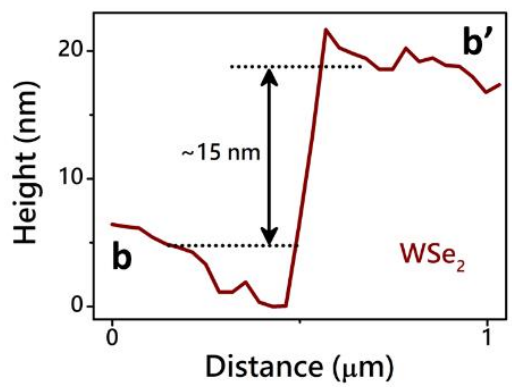

Figure S1: (a) AFM scan for the hBN flake. (b) Linescan along aa' direction shows the hBN flake thickness to be $\sim 35 \mathrm{~nm}$. (c) AFM scan for the WSe 2 flake. (b) Linescan along bb' direction indicates the $\mathrm{WSe}_{2}$ flake thickness to be $\sim 15 \mathrm{~nm}$. 


\section{S2: Polarity transition in similar devices with ambipolar $\mathrm{WSe}_{2}$ and $\mathrm{MoSe}_{2}$ channels}

Polarity transition between $n$-type and $p$-type FET has been reproduced in other similar devices with ambipolar $\mathrm{WSe}_{2}$ channel as shown below.
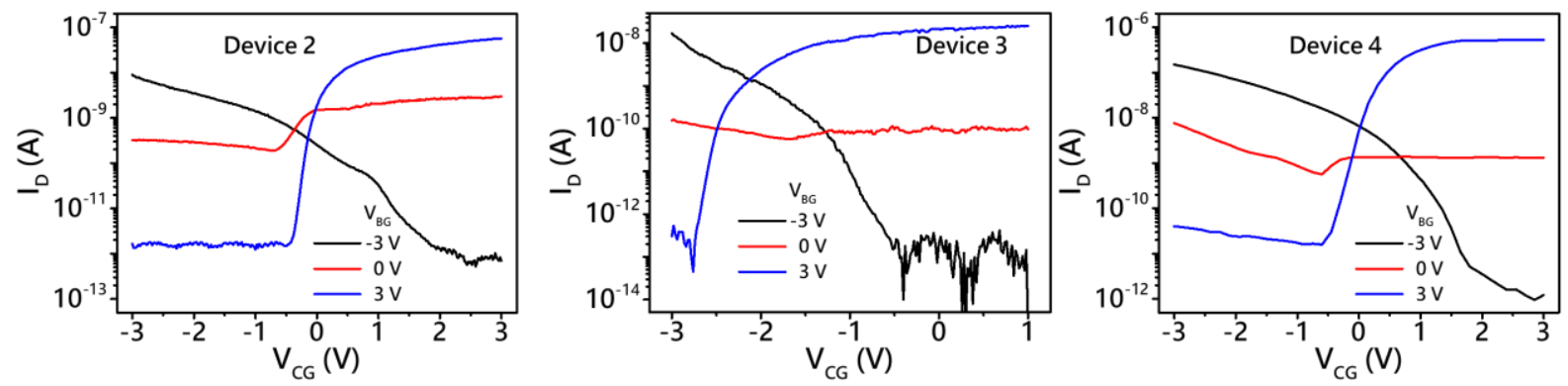

Figure S2-1: Transfer curves showing polarity transition with $V_{B G}$ in similar $\mathrm{WSe}_{2}$ channel devices. Despite the device-to-device variability likely due to variability in thickness and geometry of the flakes, transition in polarity was achieved reproducibly.

(a)

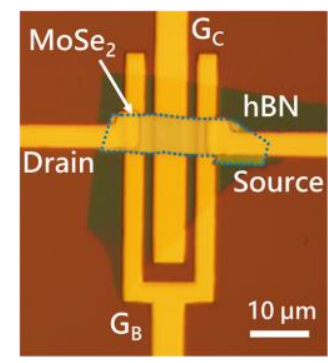

(c)

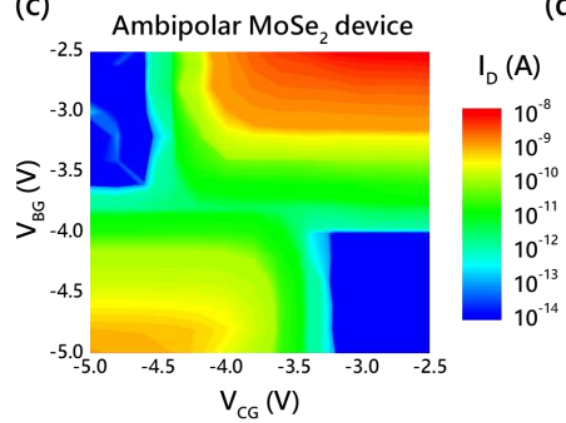

(b)

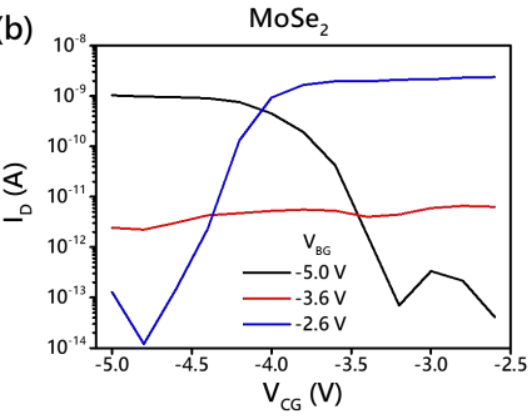

(d)

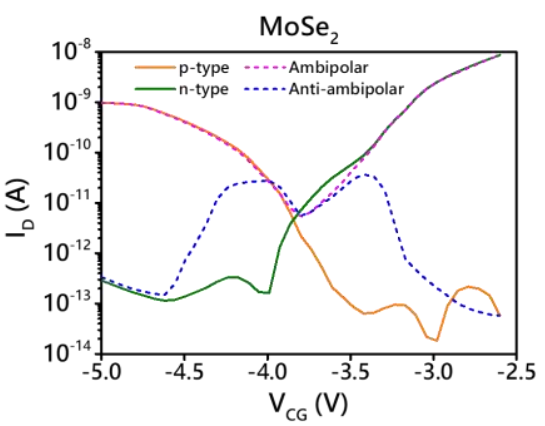

Figure S2-2: (a) Optical image of a similar device with $\mathrm{MoSe}_{2}$ as channel material. (b) Polarity transition with barrier gate-bias in ambipolar $\mathrm{MoSe}_{2}$ similar to the $\mathrm{WSe}_{2}$ devices was observed. (c) Current map of the device for varying gate bias values. A larger $n$-type current was recorded as compared to the $p$-type current. (d) Current traces for the four FET modes extracted from the current map in (c). 
Figure S2-2 shows how other ambipolar materials (here $\mathrm{MoSe}_{2}$ ) can give similar device operation with the proposed biasing schemes. The only requirement is the ability of polarity-control in an ambipolar material. As listed below, various reports ${ }^{1-3}$ have shown polarity-control in a range of material families which can be good candidates for such applications.

1. 2D materials: J. Chen et al. Reconfigurable MoTe $\mathrm{H}_{2}$ Field-Effect Transistors and its Application in Compact CMOS Circuits. IEEE Transactions on Electron Devices, 68, 4748 (2021).

2. Organic materials: H. Yoo et al. Reconfigurable Complementary Logic Circuits with Ambipolar Organic Transistors. Scientific Reports 6, 35585 (2016).

3. Silicon-on-insulator: L. Yojo et al. Reconfigurable Back Enhanced (BE) SOI MOSFET Used to Build a Logic Inverter. 32 ${ }^{\text {nd }}$ Symposium on Microelectronics Technology and Devices (SBMicro), 2017. 


\section{S3: Output characteristics, estimation of interface trap density and scope for device performance improvement}
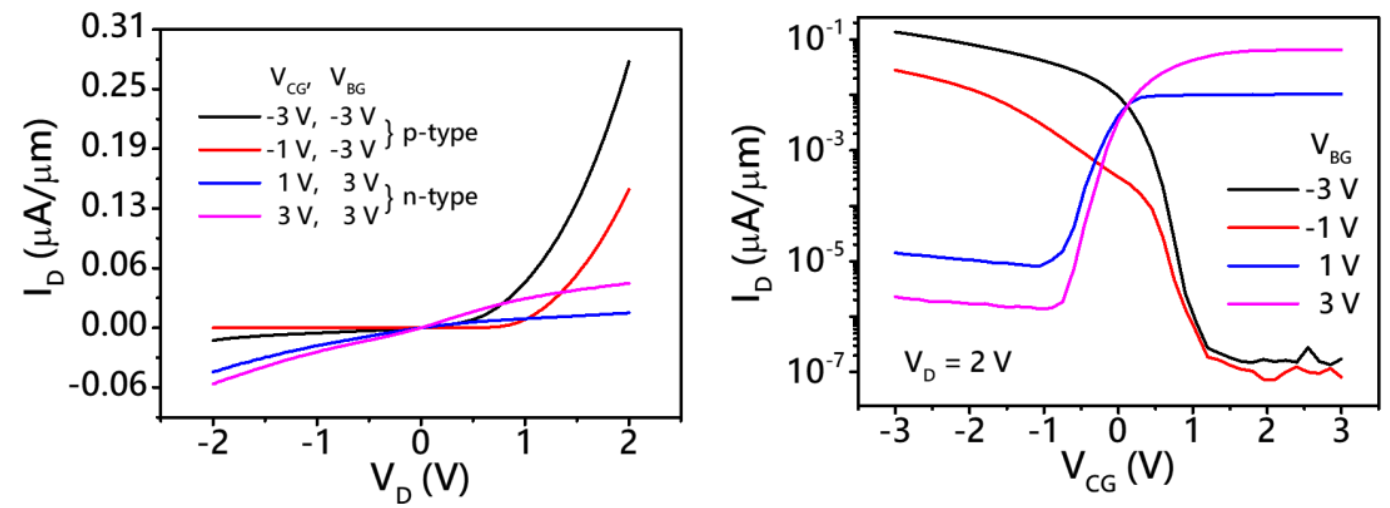

Figure S3: Output and transfer curves for the device. Rest of the data is shown in Figure 2 in the main manuscript.

Since the device relies on Schottky barrier control, the output characteristics are asymmetric due to imbalance in local band bending near the source/drain contacts under different polarities of the contact bias $\left(V_{D}\right)$.

\section{Interface Trap Density:}

The subthreshold slopes for $p$-type and $n$-type transfer curves are $\sim 276 \mathrm{mV} /$ decade and $\sim 290$ $\mathrm{mV} /$ decade, respectively. An approximate value of $280 \mathrm{mV} /$ decade is used for the following calculations.

Considering a fully-depleted $\mathrm{WSe}_{2}$ channel of $15 \mathrm{~nm}$ on top of a $35 \mathrm{~nm} \mathrm{hBN}$,

$$
\text { Subthreshold slope }=60 *\left(1+\frac{C_{D}+C_{i t}}{C_{o x}}\right) \sim 280 \mathrm{mV} / \mathrm{dec} \text { ade, }
$$

where $\mathrm{C}_{\mathrm{D}}=\epsilon_{\text {Wse } 2} \epsilon_{0} / d_{\text {Wse } 2} \approx 8 * 8.85 \times 10^{-12} / 15 \times 10^{-9}=4.72 \times 10^{-3} \mathrm{~F} / \mathrm{m}^{2}, \mathrm{C}_{\mathrm{it}}=\mathrm{q}^{2} \mathrm{D}_{\mathrm{it}}$, and

$$
\mathrm{C}_{\mathrm{ox}}=\epsilon_{h B N} \epsilon_{0} / d_{h B N} \approx 7 * 8.85 \times 10^{-12} / 35 \times 10^{-9}=1.77 \times 10^{-3} \mathrm{~F} / \mathrm{m}^{2}
$$

Substituting these values in the above equation, we get $D_{\text {it }} \sim 1.1 \times 10^{12} \mathrm{~cm}^{-2} \mathrm{eV}^{-1}$.

This rough estimate $\left(D_{\text {it }} \sim 1.1 \times 10^{12} \mathrm{~cm}^{-2} \mathrm{eV}^{-1}\right)$ agrees well with a detailed study on traps at the $\mathrm{hBN} / \mathrm{WSe} \mathrm{in}_{2}$ interface for ambipolar (8-20 nm thick) $\mathrm{WSe}_{2}$ flakes and an effectively similar device structure. ${ }^{4}$ The same study also concludes that the traps at the hBN/WSe 2 interface play an important role in polarity transition in ambipolar $\mathrm{WSe}_{2}$. 


\section{Scope for device performance improvement:}

\section{Contact resistance:}

Contact resistance values extracted by the $\mathrm{Y}$-function method $^{5}$ are $390 \mathrm{k} \Omega \cdot \mu \mathrm{m}$ and $630 \mathrm{k} \Omega \cdot \mu \mathrm{m}$ for $p$-type and $n$-type FETs, respectively. According to a general estimate for metal contacts with TMDs, ${ }^{6}$ contact resistance values can be brought down to $1-10 \mathrm{k} \Omega \cdot \mu \mathrm{m}$. Assuming that effective carrier mobility increases proportionally with reduction in contact resistance, the device response time can be scaled by approximately $100 \times$ with proper contact engineering.

\section{Chemical doping:}

Several studies have demonstrated both in situ and post-fabrication chemical doping for $\mathrm{WSe}_{2}$ for

controllable $n$-type or $p$-type transport enhancement. ${ }^{7,8}$ Chemical doping, while carefully preserving the ambipolar transport, could be implemented to improve channel sheet resistance. Further, selective doping near contacts could also help reduce contact resistance and lead to ohmiclike behaviour.

\section{Device architecture:}

Devices in this work have a separation of $\sim 1.5-2 \mu \mathrm{m}$ between $\mathrm{G}_{\mathrm{C}}$ and $\mathrm{G}_{\mathrm{B}} \mathrm{S}$. The resulting large area of ungated $\mathrm{WSe}_{2}$ channel region can limit device current levels due to high sheet resistance, and could be improved easily by appropriate gate electrode patterning. ${ }^{9}$ 
S4: Transfer curves by interchanging $V_{C G}$ and $V_{B G}$

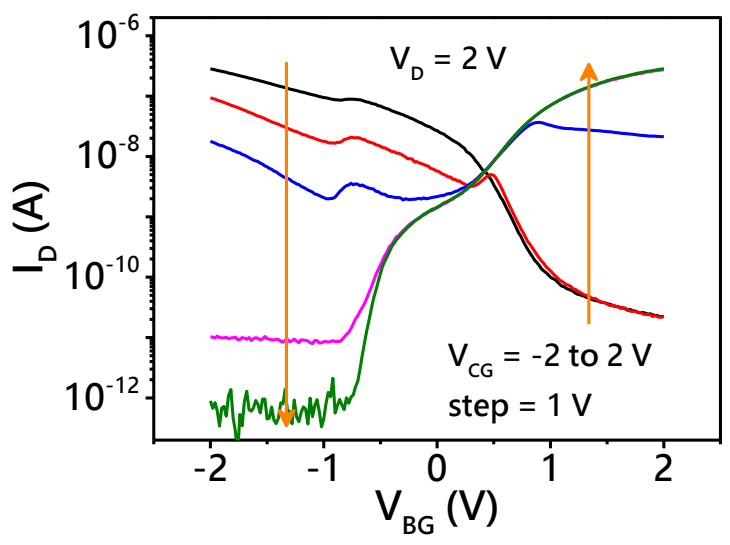

Figure S4: Transfer curves by sweeping $V_{B G}$ as primary variable for varying $V_{C G}$.

The bump is likely due to a combined contribution from the following factors.

(i) Body current in thin-body FET (similar to silicon on insulator, or SOI)

(ii) Mismatch between Schottky barrier and channel barrier in equilibrium

(iii) Band bending in the intrinsic channel area between $G_{B} S$ and $G_{C}$. 
S5: Photoresponse of the device for a wide range of optical power ( $1 \mathrm{nW}$ to $1 \mu \mathrm{W}$ )
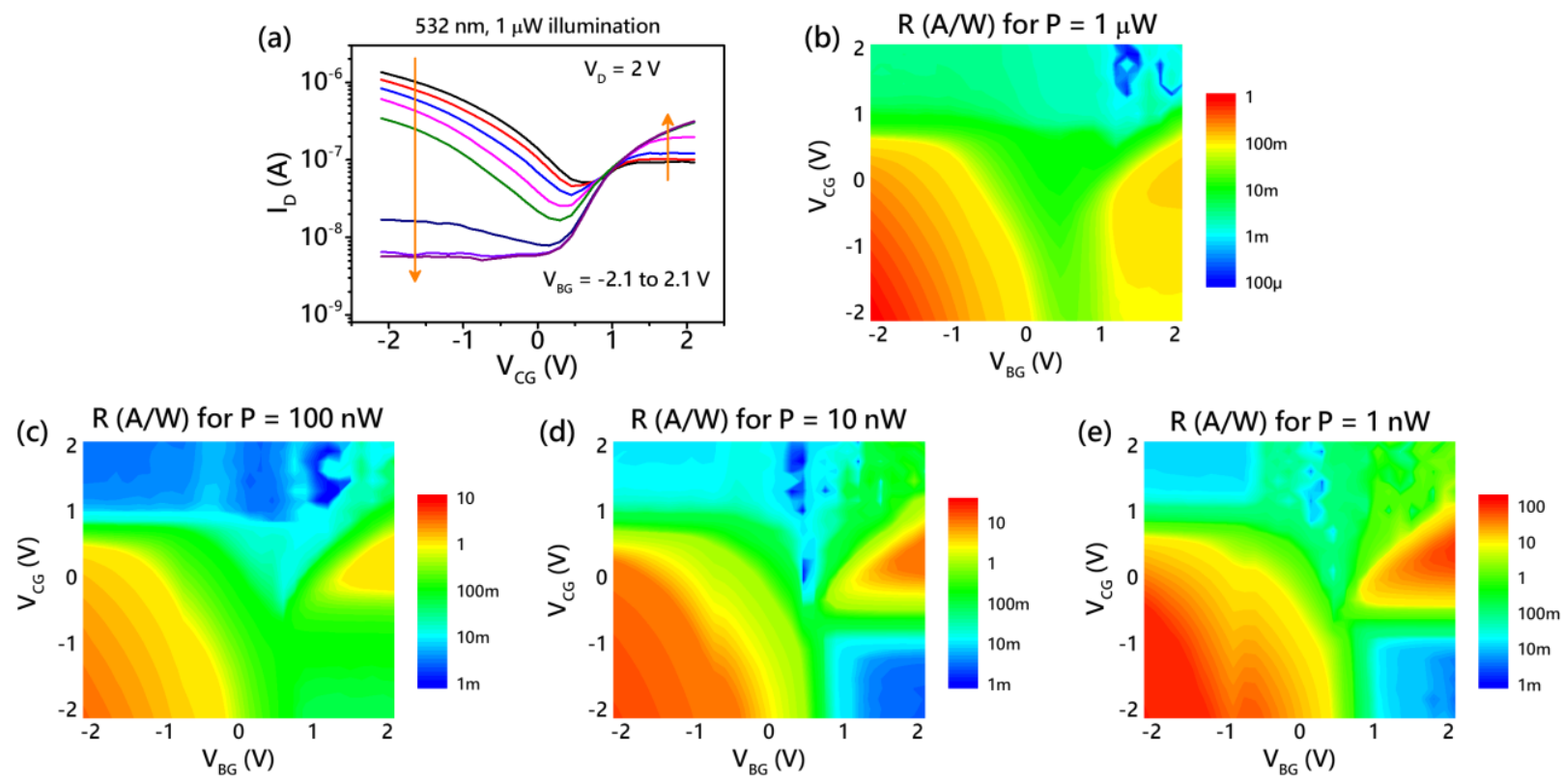

Figure S5-1: (a) Transfer curves of the $\mathrm{WSe}_{2} \mathrm{FET}$ with varying $V_{B G}$ values under $532 \mathrm{~nm}, 1 \mu \mathrm{W}$ focused beam illumination. (b) Responsivity values as a function of the two gate biases extracted from (a) and dark current shown in Figure 2a in the main manuscript calculated as $R=\left(I_{\text {illum }}-\right.$ $I_{\text {dark }}$ )/ P. Similarly, calculated responsivity values for (c) $100 \mathrm{nW}$, (d) $10 \mathrm{nW}$, and (e) $1 \mathrm{nW}$ of $532 \mathrm{~nm}$ laser illumination. Responsivity values as high as $>180 \mathrm{~A} / \mathrm{W}$ were obtained for $1 \mathrm{nW}$ optical power. 

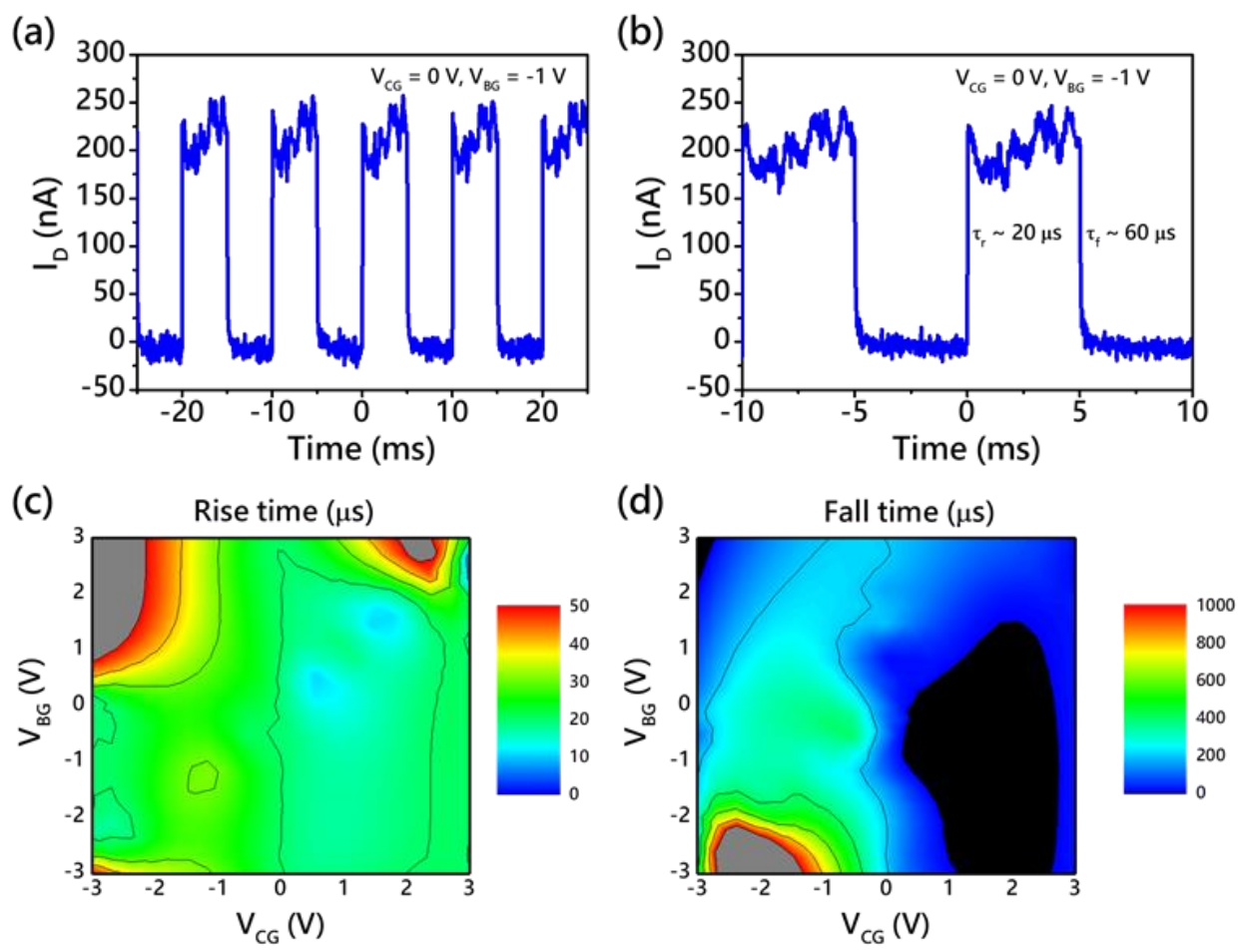

Figure S5-2: (a) An example of photocurrent switching measurement under $1 \mu \mathrm{W}$ of $532 \mathrm{~nm}$ laser for fixed gate-bias values. Such scans are repeated for varying gate bias. (b) High-resolution scan of photocurrent switching for the same bias as in (a). 2D color maps for (c) rise time $\left(\tau_{r}\right)$ and (d) fall time $\left(\tau_{f}\right)$ extracted as shown in (b). Grey and black regions indicate unavailability of data. It can be observed that the majority of bias combinations result in fall times smaller than $0.5 \mathrm{~ms}$. 
S6: FFT Analysis of the ac waveforms
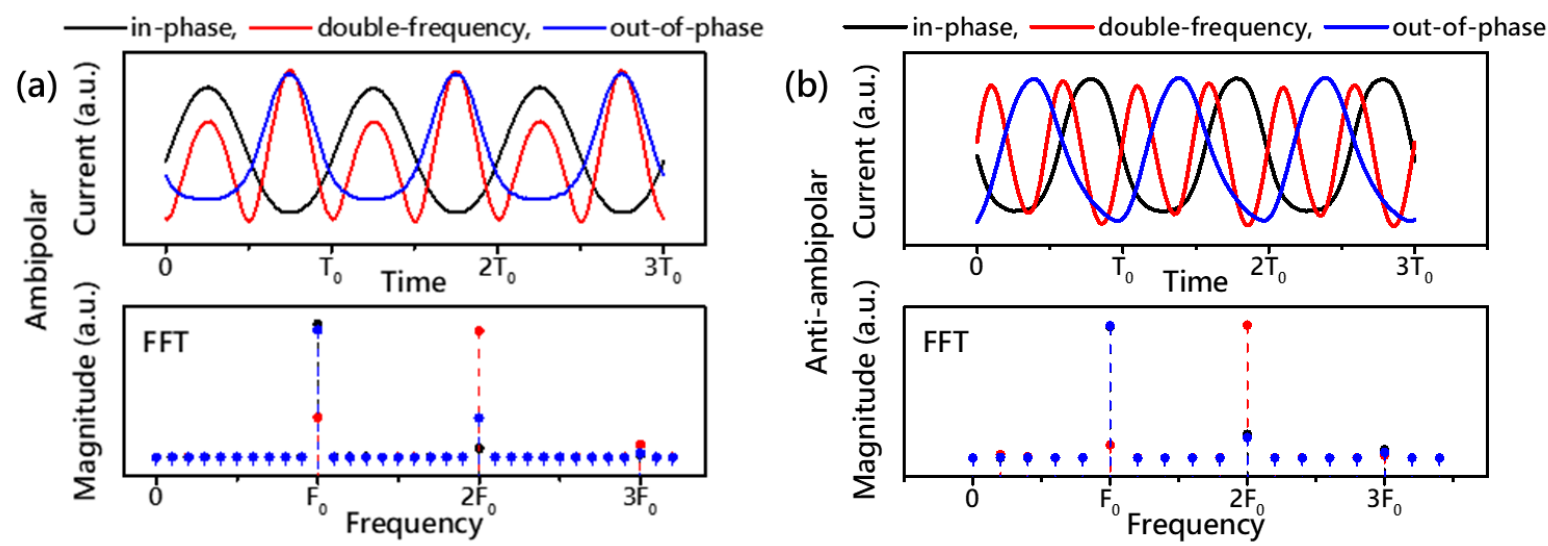

Figure S6: Recorded ac waveforms (top panel) and their corresponding FFT magnitude (bottom panel) for (a) ambipolar, and (b) anti-ambipolar behavior. FFT results show negligible magnitude after the $2^{\text {nd }}$ harmonic frequency. 


\section{S7: Hysteresis and variability in device operation}
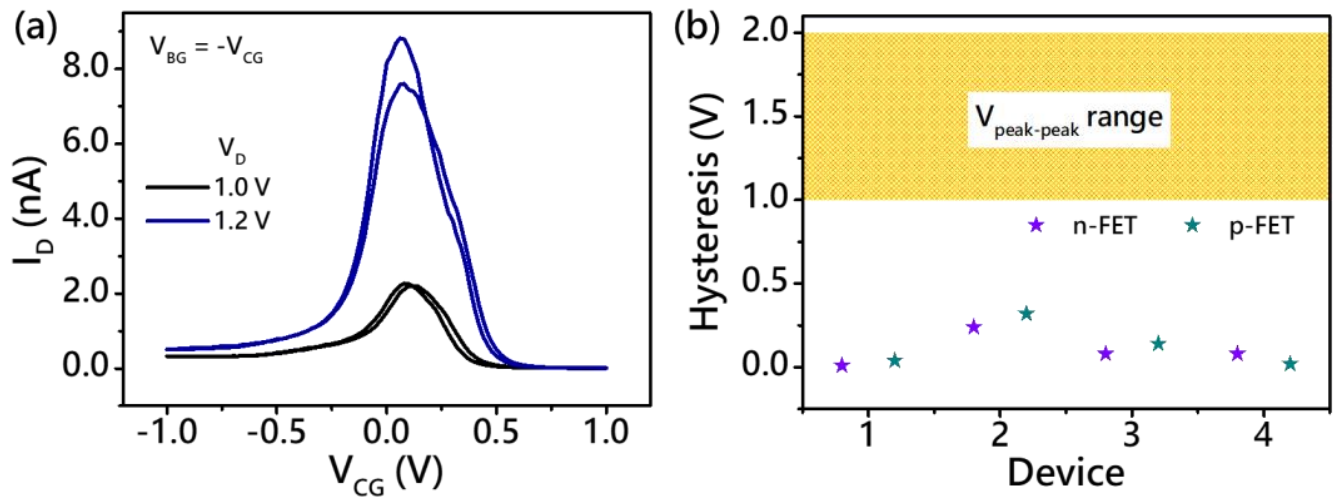

Figure S7-1: (a) Negligible hysteresis in anti-ambipolar transfer characteristics suggests good reliability in ac waveforms. (b) Hysteresis values measured at constant current for several devices as compared to the applied gate-bias range suggest that their effect on the ac device operation would be minimal.

Device parameters such as carrier mobility, hysteresis, and cross-over voltage $\left(V_{C P}\right)$ were extracted for the four $\mathrm{WSe}_{2}$ devices. Their distributions (or variation) are plotted below.
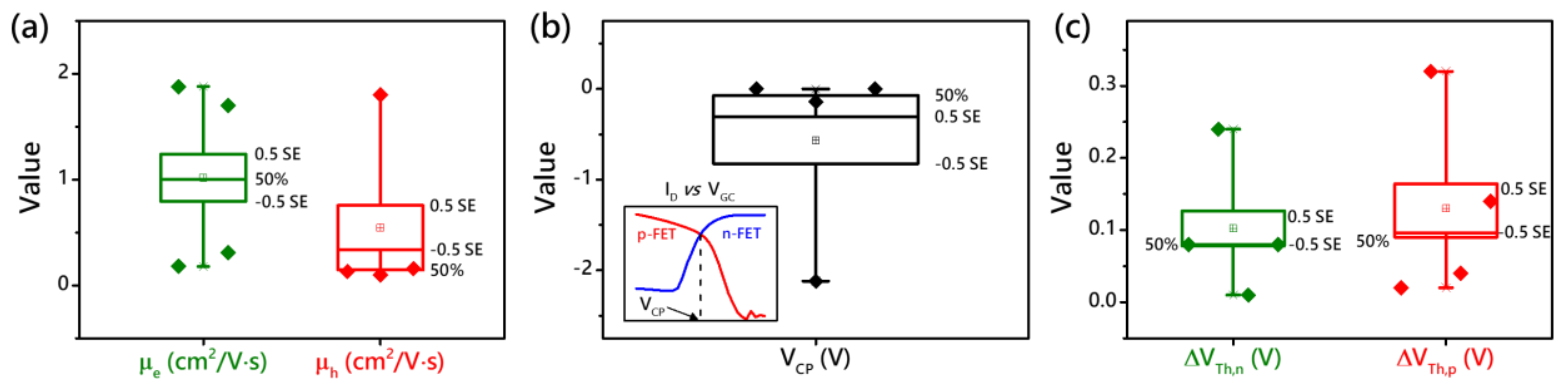

Figure 7-2: Variation in (a) carrier mobilities, (b) crossover voltage $\left(V_{C P}\right)$, and (c) hysteresis from four $\mathrm{WSe}_{2}$ transistors has been shown. SE denotes standard error.

Device-to-device variation observed here can be mainly attributed to arbitrary shapes and thicknesses of mechanically exfoliated flakes (both $\mathrm{WSe}_{2}$ and $\mathrm{hBN}$ ). Use of large area grown 2D materials can help alleviate device-to-device variability issues and enable scalable manufacturing.

Variability across measurements is also important from device performance and reliability point of view. This is even more relevant since the photo-illumination can induce long term instability 
in dark-state currents. Variability across measurements was probed by systematic data acquisition and analysis as shown in the figure below.
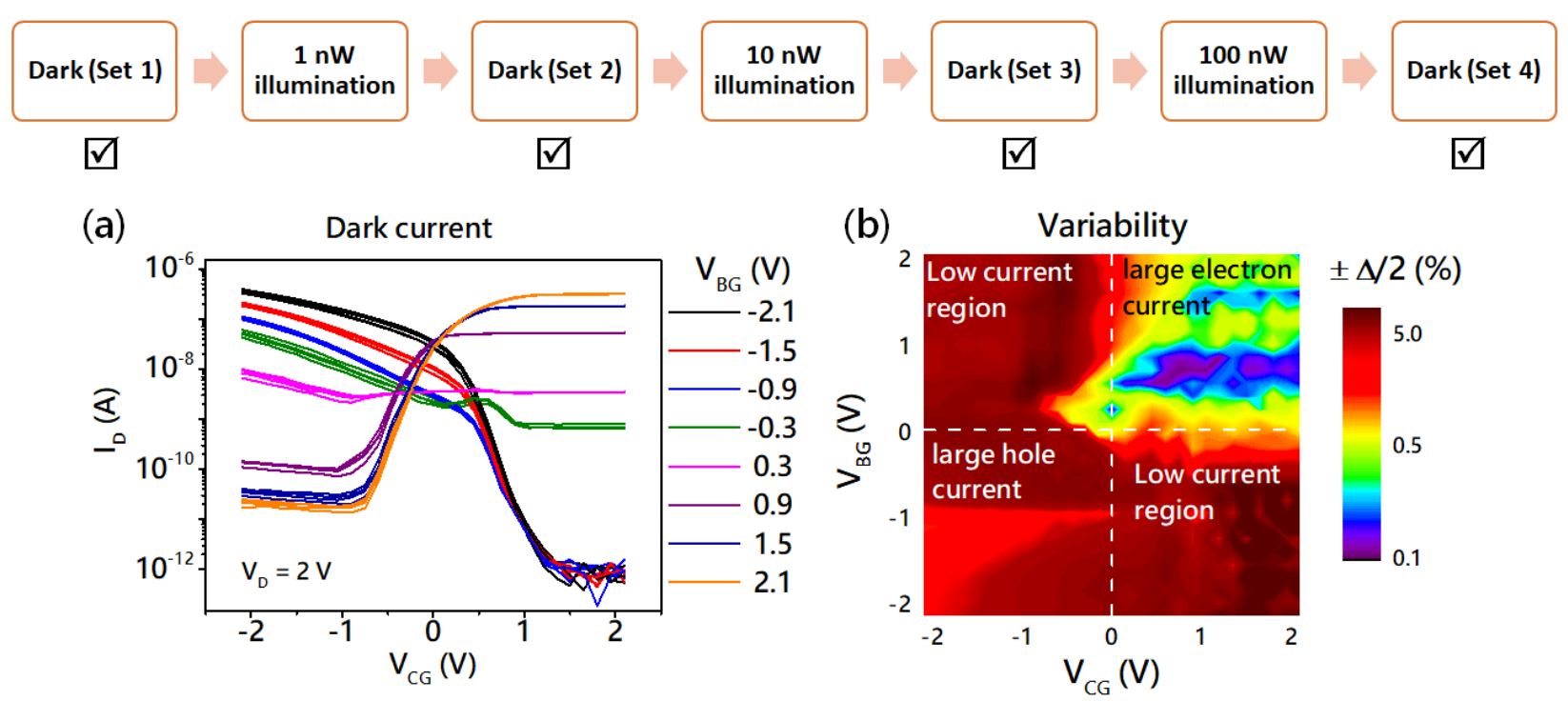

Figure 7-3: Top panel shows the data acquisition steps. Dark state device current was used to evaluate the variability in device operation. (a) Four sets of dark state transfer curves for varying barrier gate bias. (b) Colour map for the relative spread $( \pm \Delta / 2)$ of the dark current values represented by a ratio of standard deviation to the mean current value $\left(\Delta=\sigma_{i} / \mu_{i}\right)$ for each biasing point.

Each step in the top panel of Figure S7-3 indicates recording of a full current map for whole range of gate biases. Each recording took roughly 7 minutes, and the whole sequence was completed in approximately 50 minutes (3000 seconds). Furthermore, increasing illumination ( $1 \mathrm{nW}$ to $100 \mathrm{nW}$ ) also ensured that any long-term operational variability induced by exposing $\mathrm{WSe}_{2}$ channel to light is accounted for. Figure 7-3(a) shows the dark state transfer curves. The spread in current values is relatively narrow. To further analyse the data, standard deviation $\left(\sigma_{i}\right)$ and mean $\left(\mu_{i}\right)$ of current values at each biasing point were determined and their ratio $\left(\Delta=\sigma_{i} / \mu_{i}\right)$ was used to determine relative spread of the current values around the average value. A small error $( \pm \Delta / 2)$ of $\sim 6 \%$ was observed as shown in Figure 7-3(b) suggesting reliable operation. It must be noted that device current variation in low current regions (as marked in the figure) is less important because of too low current values. 
S8: AC switching data at $1 \mathrm{kHz}$ signal for anti-ambipolar mode of operation

\section{Output current switching for $1 \mathrm{kHz}$ gate signal}

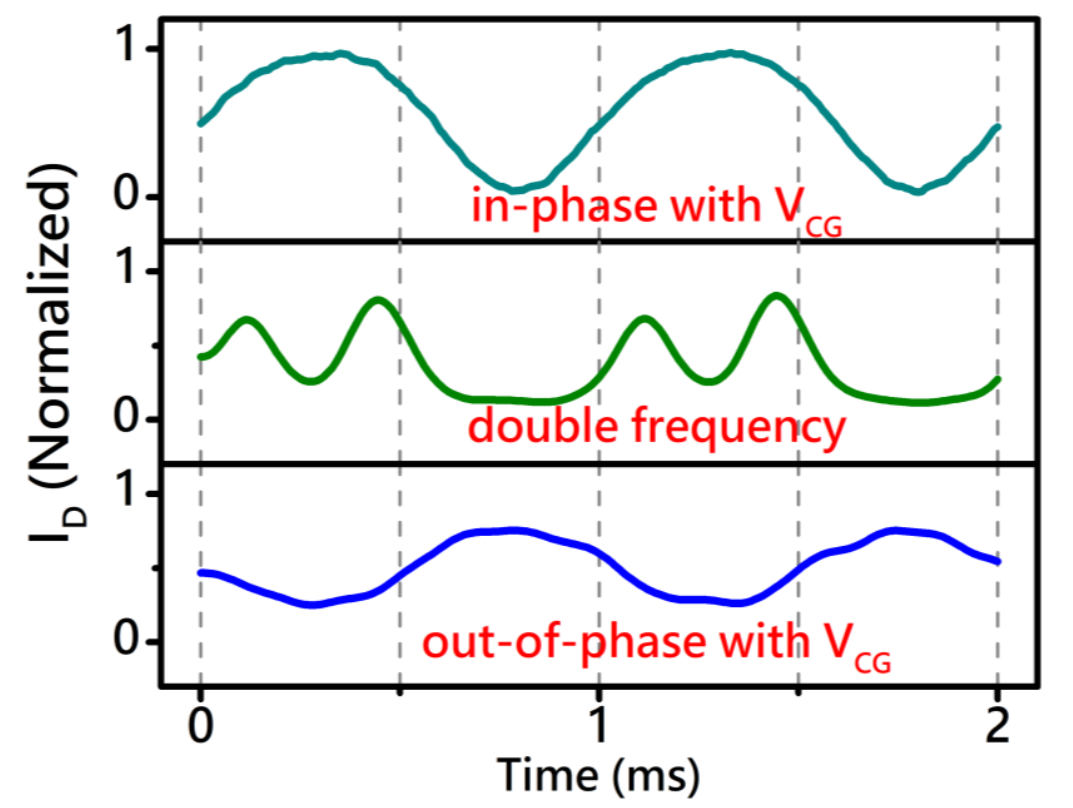

Figure S8: In-phase, double-frequency and out-of-phase current waveforms generated under antiambipolar mode of operation by applying $180^{\circ}$ shifted $1 \mathrm{kHz}$ sinewaves at the two gate contacts. 
S9: Left shift in anti-ambipolar peak under illumination

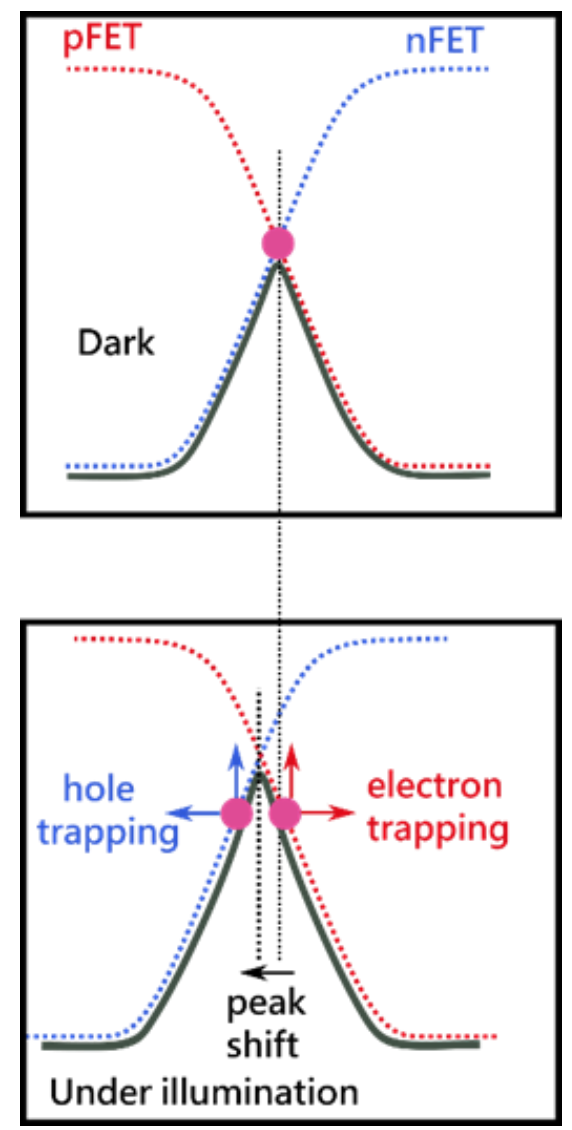

Figure S9: Illustration showing how a left shift in anti-ambipolar peak is possible under illumination due trapping of minority carriers. The direction and magnitude of shift is decided by the number of trapped electrons and holes. 
S10: Real-time frequency switching showing a frequency shift with the laser

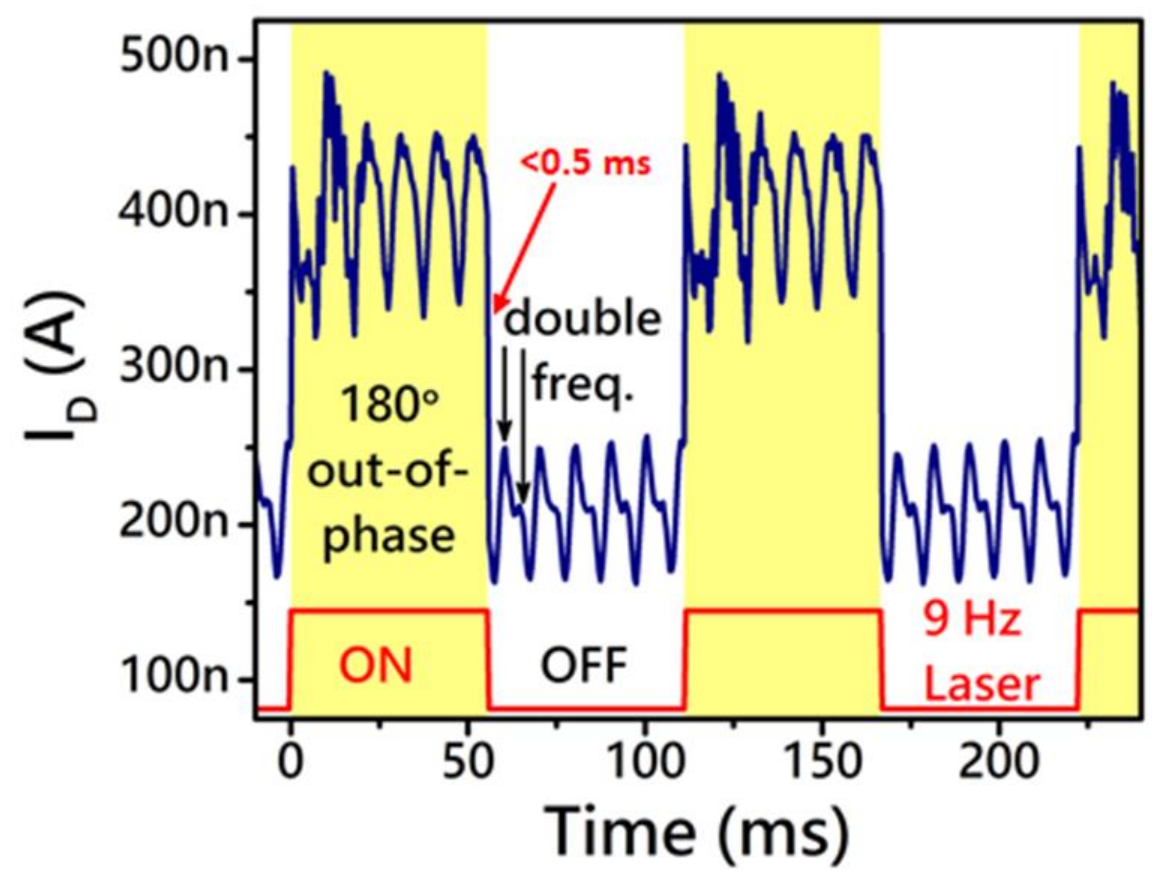

Figure S10: Output current waveform showing synchronized integration of laser input along with ac signals applied at gate electrodes to demonstrate real-time frequency switching with fast response time $(<0.5 \mathrm{~ms})$. 
S11: Metrics and $E$ values for 2-bit schemes
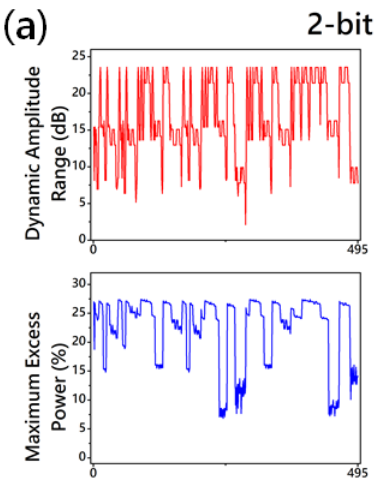

-bit schemes
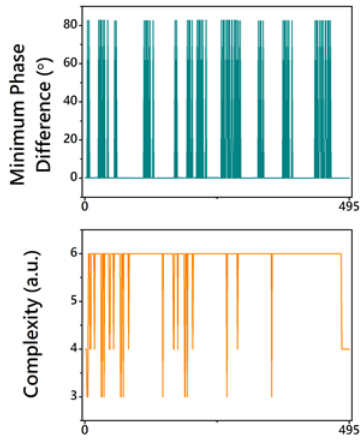

(b)

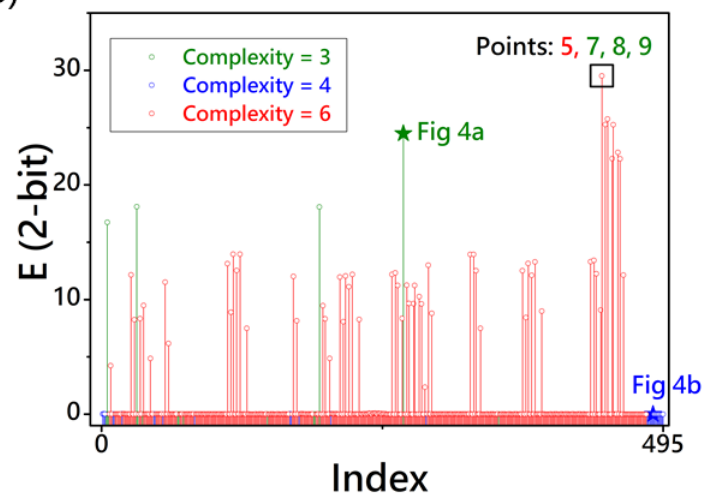

Figure S11: Values of the four performance metrics for all possible (a) 2-bit schemes from the symbol space shown in Figure 5b in the main manuscript. (b) $E$ values for all possible schemes calculated from their metrics in (a). 


\section{S12: Performance evaluation of 3-bit schemes}
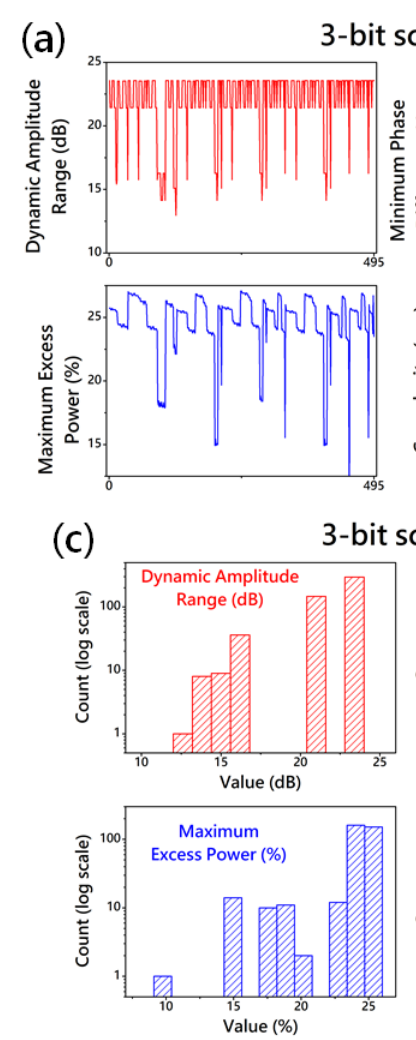

(e)
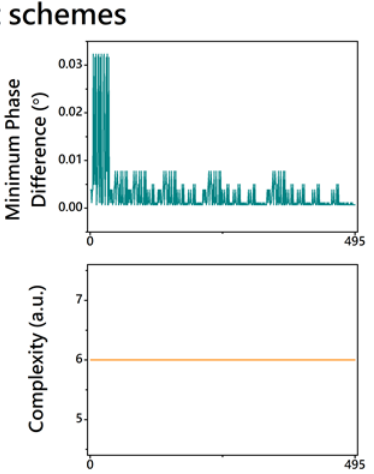

bit schemes
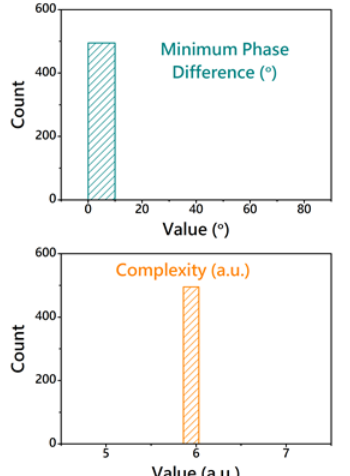

(b)

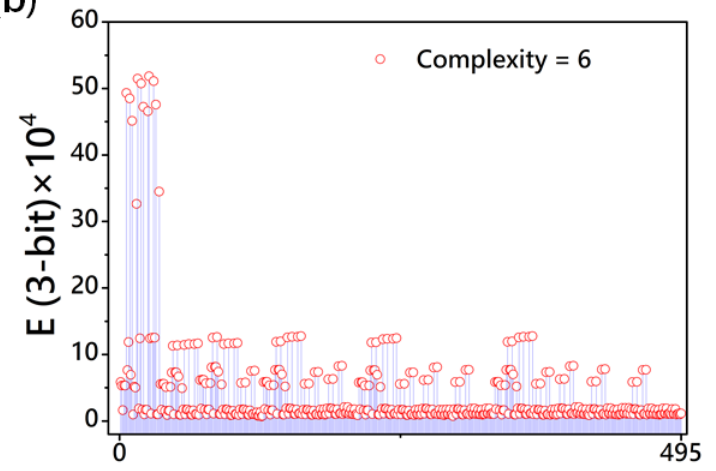

(d)

Index

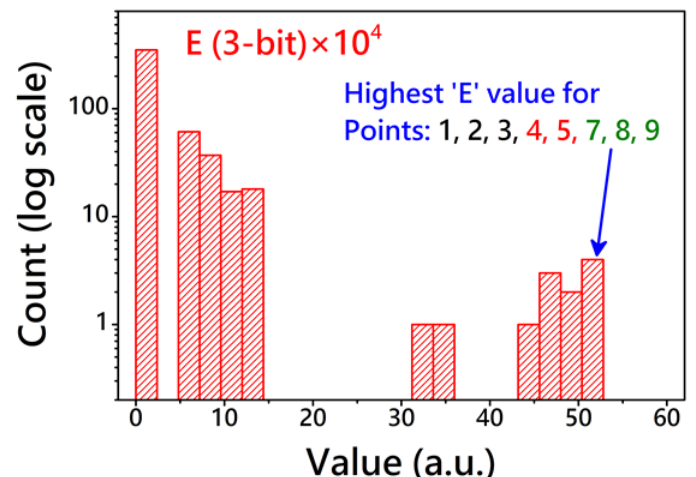

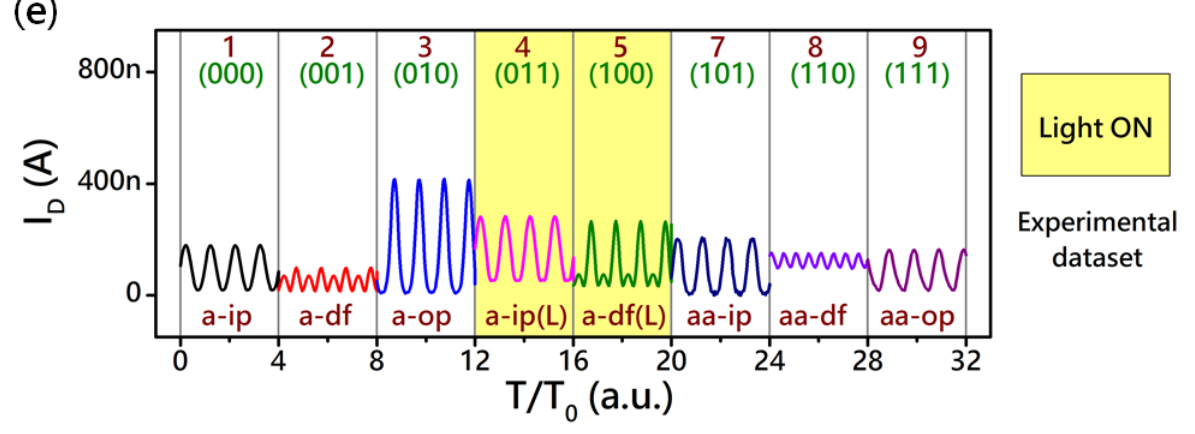

Figure S12: Values of the four performance metrics for all possible (a) 3-bit schemes from the symbol space shown in Figure 5b in the main manuscript. (b) $E$ values for all possible schemes calculated from their metrics in (a). Histograms of (c) the four performance metrics, and (d) $E$ for the metric values from (a) and (b), respectively. It should be noted that all 3-bit cases have minimum MPD and highest hardware complexity values, and $E$ is mainly determined by DAR and MEP performance. (e) Collated output current waveforms corresponding to the case with the highest $E$ value mentioned in (d). Please refer to Figure $5 \mathrm{~b}$ in the main manuscript for the symbol space. 


\section{S13: Performance evaluation for a symbol space of 20 points}

To compare all possible combinations of the cases where 2-bit encoding may be implemented with or without the need of switching between ambipolar and anti-ambipolar modes, a similar evaluation as described in Fig 5 in the main manuscript has been carried out for an extended symbol space of 20 points. The selected biasing points (similar to Fig 5a) and evaluation of 2-bit encoding schemes (similar to Fig 5f) without a distinction with regards to use of illumination are as shown in the figure below.
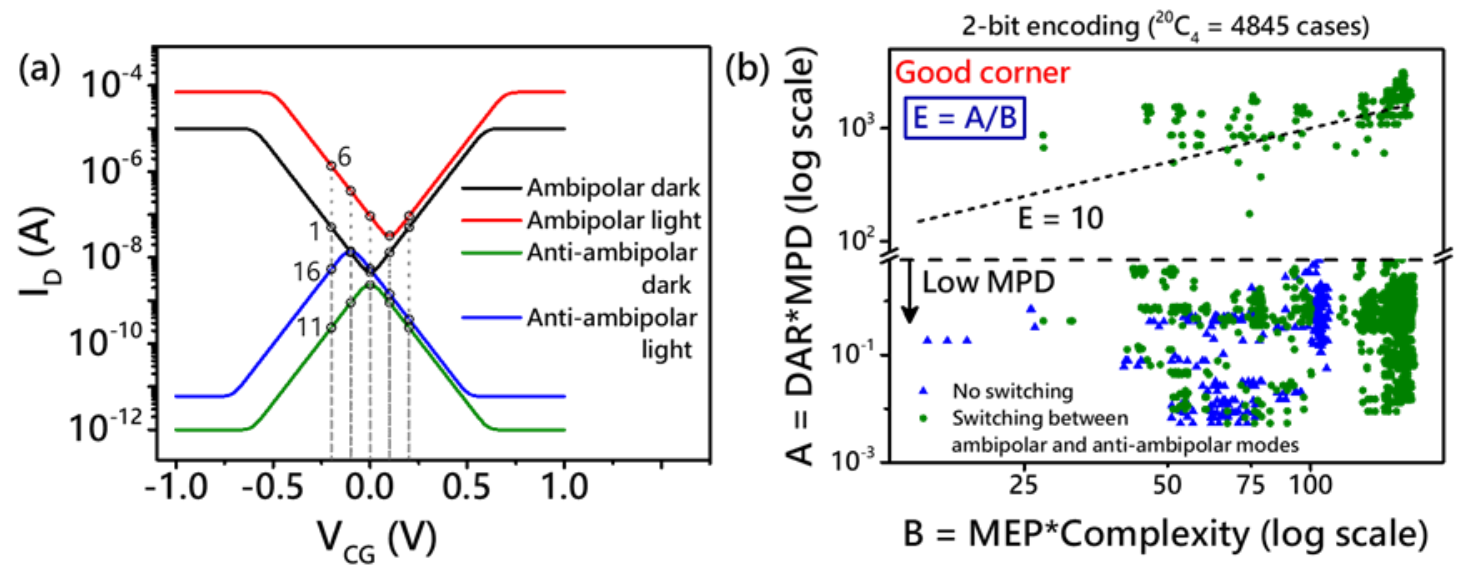

Figure S13: (a) Simulated ideal transfer curves for ambipolar and anti-ambipolar channel transport, under dark and illuminated (light) conditions. The points highlighted by black circles are used for further analysis. The first point in each curve is numbered for identification. (b) Scatter plot showing $\mathrm{A}=\mathrm{DAR} * \mathrm{MPD}$ vs $\mathrm{B}=\mathrm{MEP} *$ Complexity for all 2-bit encoding schemes. $\mathrm{A}$ distinction is made among the cases with and without switching between ambipolar and antiambipolar modes. Evaluation parameter can be calculated as $E=\mathrm{A} / \mathrm{B}$. The points with low value of A suffer from low MPD. $E=10$ line is shown as a reference. Points farther away from this line on the top have higher $E$ values.

The data reinforces the argument that the cases in which switching between ambipolar and antiambipolar modes is included can outperform the cases where it is absent. 


\section{References}

[1] Chen, J.; Li, P.; Zhu, J.; Wu, X.-M.; Liu, R.; Wan, J.; Ren, T.-L. Reconfigurable Mote2 Field-Effect Transistors and Its Application in Compact Cmos Circuits. IEEE Transactions on Electron Devices, 2021, 68, 4748.

[2] Yoo, H.; Ghittorelli, M.; Smits, E. C. P.; Gelinck, G. H.; Lee, H.-K.; Torricelli, F.; Kim, J.-J. Reconfigurable Complementary Logic Circuits with Ambipolar Organic Transistors. Scientific Reports, 2016, 6, 35585.

[3] Yojo, L.; Rangel, R. C.; Sasaki, K. R. A.; Martino, J. A. Reconfigurable Back Enhanced (Be) Soi Mosfet Used to Build a Logic Inverter. 2017 32nd Symposium on Microelectronics Technology and Devices (SBMicro), 2017.

[4] Ali, F.; Ahmed, F.; Taqi, M.; Mitta, S. B.; Ngo, T. D.; Eom, D. J.; Watanabe, K.; Taniguchi, T.; Kim, H.; Hwang, E.; Yoo, W. J. Traps at the Hbn/Wse2 Interface and Their Impact on Polarity Transition in Wse2. 2D Materials, 2021, 8, 035027.

[5] Park, J. Y.; Joe, H.-E.; Yoon, H. S.; Yoo, S.; Kim, T.; Kang, K.; Min, B.-K.; Jun, S. C. Contact Effect of Res2/Metal Interface. ACS Applied Materials \& Interfaces, 2017, 9, 26325.

[6] Jena, D.; Banerjee, K.; Xing, G. H. Intimate Contacts. Nature Materials, 2014, 13, 1076.

[7] Fang, H.; Chuang, S.; Chang, T. C.; Takei, K.; Takahashi, T.; Javey, A. High-Performance Single Layered Wse2 P-Fets with Chemically Doped Contacts. Nano Letters, 2012, 12, 3788.

[8] Ji, H. G.; Solís-Fernández, P.; Yoshimura, D.; Maruyama, M.; Endo, T.; Miyata, Y.; Okada, S.; Ago, H. Chemically Tuned $P$ - and $N$-Type $W_{s e}$ Monolayers with High Carrier Mobility for Advanced Electronics. Advanced Materials, 2019, 31, 1903613.

[9] Müller, M. R.; Salazar, R.; Fathipour, S.; Xu, H.; Kallis, K.; Künzelmann, U.; Seabaugh, A.; Appenzeller, J.; Knoch, J. Gate-Controlled Wse2 Transistors Using a Buried Triple-Gate Structure. Nanoscale Research Letters, 2016, 11, 512. 